\title{
Revisiting Simple Schizophrenia - The Search (in the absence) of Answers in Current Time
}

Borges, J.; Macedo, P.; Leite, R.; Dias dos Santos, G.; Costa, A. L.; Vicente, C. Department of Psychiatric and Mental Health of Centro Hospitalar do Baixo Vouga, Aveiro - Portugal

\section{Introduction}

By revisiting classical nosology, the present case study intends to address less quantifiable aspects of mental illness. The heterogeneity in the presentation and course of schizophrenia spectrum disorders has always been a challenge in diagnosis, therapy and rehabilitation.

\section{Background}

The concept of schizophrenia is relatively recent compared to other mental disorders described since ancient times. In France, Morel (1809-1873) referred to cases of Early Dementia, in Scotland, Clouston (1840-1915) coined the term Teenage Insanity and in Germany emerged terms such as Catatonic Syndrome with Kahlbaum or Hebephrenia with Hecker. Emil Kraeplin (1856-1926) integrated the various clinical pictures by organizing them nosologically under the name of Dementia Praecox and later, Bleuler (1857-1939), defined the entity still known today as Schizophrenia. The motivation for the publication of this clinical case is precisely to revisit in the eyes of classical psychiatry, Simple Schizophrenia (SS), a heterogeneous entity of difficult consensus in its classification.

We describe the case of a young man with normative life course and exemplary school performance who underwent an insidious cut in his function coinciding with the entrance into college and cannabinoid consumption. Interestingly he never had demonstrated obvious psychotic symptoms. Several diagnostic hypotheses can be put such as SS and Amotivational Syndrome (AS).

\section{Clinical Case}

Male, 23 years old. Caucasian. Single. Lives with parents and brother.

Father: Accountant.

Mother: Elementary School Teacher. Occupation: $2^{\text {nd }}$ year of Civil

Engineering student since 5 years ago.

\section{Discussion}

SS can currently only be diagnosed in the absence of other conditions. In this case, this does not seem likely to be, given the patient's age and his pre-morbid functioning. Another of the verified features is the absence of anomalous subjective experiences related to another psychotic disturbance. Historically, the concept of SS was originated in 1903 by Otto Diem who described it as a subgroup of Kraeplin's Early Dementia. Later, Bleuler, in 1908, integrated the term as a syndrome, considering in it the fundamental symptoms of schizophrenia and the absence of accessory symptoms. In 1913, Kraeplin also recognized it. These three authors agreed that SS, as found in this case, is characterized by personality change, loss of interest and loss of functional capacity. The diagnosis was generally accepted and was incorporated into the ICD classifications in 1948 and DSM-I in 1952. The concept has disappeared in the DSM-III and, although we still find it in ICD-10, its authors recommend its use with caution.

The Amotivational Syndrome is not included in the current classifications. Despite being referred to in DSM-5 only as a possible consequence of chronic cannabis use, there are no clear diagnostic criteria. This syndrome is essentially characterized by apathy, affective dullness and decreased motivation. Although in this case there are some of this characteristics, it seems that during periods of abstinence, the alterations observed are more than a state of acute intoxication and less than a toxic psychotic state, with the particularity of symptomatic permanence in time and neurocognitive impairment.

\section{Conclusions}

Since the diagnosis of SS is difficult, there is necessarily difficulty in its treatment and follow-up. This picture complicates when the individual consumes substances not only due to the stigma related to additive disorders, but also by the symptomatic mix that it represents. Thus, it is important to highlight a condition which, due to its natural history, inevitably leads to significant functional deterioration. That way, early diagnosis and treatment are essential. The increasing prevalence of tetrahydrocannabinoid use in the young population may contribute significantly to mask primary psychiatric conditions.

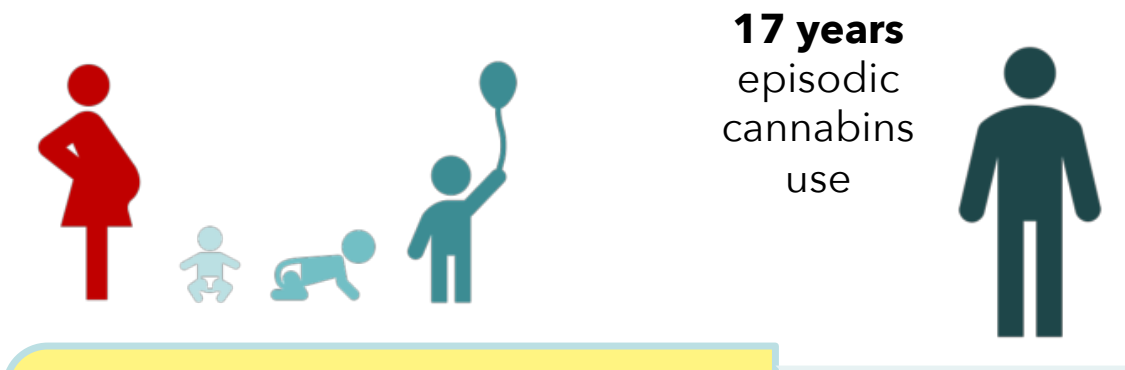

Uneventful pregnancy/eutocic delivery Normative psychomotor development. No school fails/Keen student. Preserved socialization skills.

Other Information:
Followed for 1 year, with prolonged periods of cannabis abstinence ( $>6$ months) always maintaining a clinical picture as we describe. He tried to engage undifferentiated jobs (since he does not attend college) unsuccessfully because of non-compliance with schedules and difficulty in performing the required tasks.

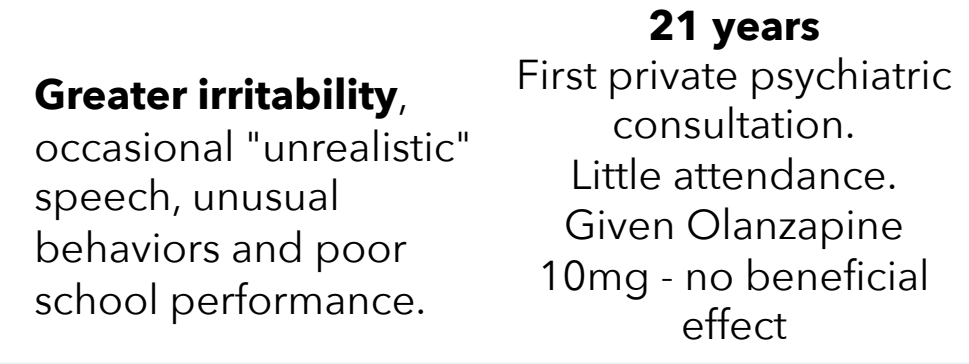
Lack of insight. Multiple complaints about his parents blaming them for his failures, admitting verbal aggression.
Normal CT Scan and EEG. Refused proposed psychometric tests (MMPI and WAIS).

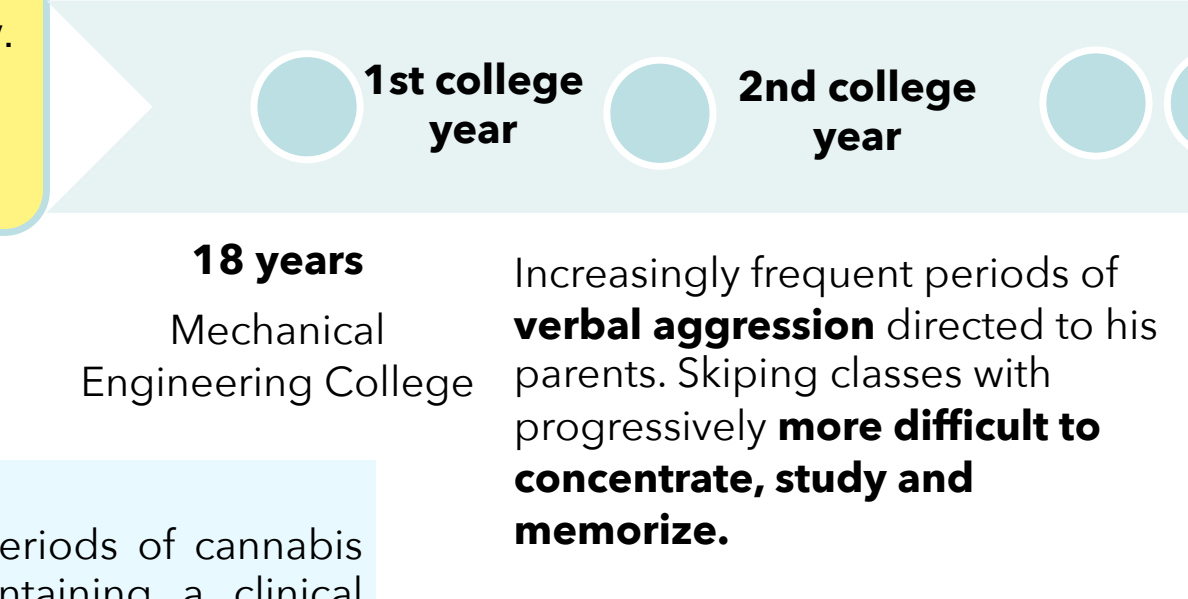

memorize.
He stays 6 months without consuming cannabis and has been given Aripiprazol $10 \mathrm{mg}$ id 